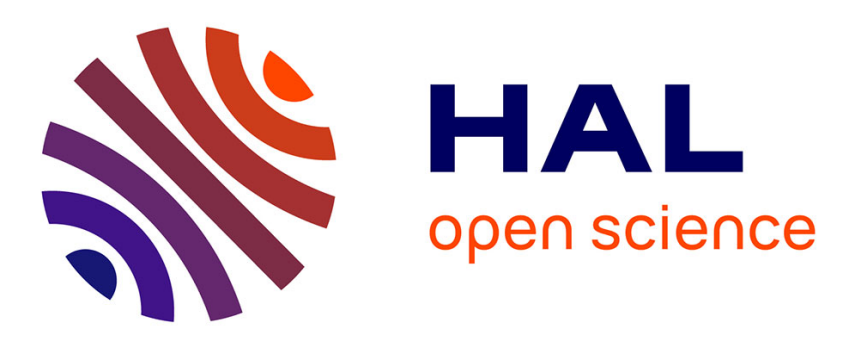

\title{
A Fine-Grained Analysis of Radar Detection in Vehicular Networks
}

Gourab Ghatak, Sanket Sanjay Kalamkar, Yash Gupta, Shubhi Sharma

\section{To cite this version:}

Gourab Ghatak, Sanket Sanjay Kalamkar, Yash Gupta, Shubhi Sharma. A Fine-Grained Analysis of Radar Detection in Vehicular Networks. IEEE GLOBECOM 2021 - IEEE Global Communications Conference, Dec 2021, Madrid, Spain. hal-03328162

\section{HAL Id: hal-03328162 https://hal.science/hal-03328162}

Submitted on 28 Aug 2021

HAL is a multi-disciplinary open access archive for the deposit and dissemination of scientific research documents, whether they are published or not. The documents may come from teaching and research institutions in France or abroad, or from public or private research centers.
L'archive ouverte pluridisciplinaire HAL, est destinée au dépôt et à la diffusion de documents scientifiques de niveau recherche, publiés ou non, émanant des établissements d'enseignement et de recherche français ou étrangers, des laboratoires publics ou privés. 


\title{
A Fine-Grained Analysis of Radar Detection in Vehicular Networks
}

\author{
Gourab Ghatak ${ }^{\dagger}$, Sanket S. Kalamkar ${ }^{\ddagger}$, Yash Gupta ${ }^{\dagger}$, and Shubhi Sharma ${ }^{\dagger}$ \\ ${ }^{\dagger}$ Department of ECE, IIIT Delhi, India ${ }^{\ddagger}$ INRIA, Paris, France \\ E-mail: gourab.ghatak@iiitd.ac.in, sanket.kalamkar@inria.fr, yash17210@iiitd.ac.in, and shubhi17195@iiitd.ac.in
}

\begin{abstract}
Automotive radar is a critical feature in advanced driver-assistance systems. It is important in enhancing vehicle safety by detecting the presence of other vehicles in the vicinity. The performance of radar detection is, however, affected by the interference from radars of other vehicles as well as the variation in the target radar cross-section (RCS) due to varying physical features of the target vehicle. Considering such interference and random RCS, this work provides a fine-grained performance analysis of radar detection. Specifically, using stochastic geometry, we calculate the meta distribution of the signal-tointerference-and-noise ratio that permits the reliability analysis of radar detection at individual vehicles. We also evaluate the delay aspect of radar detection, namely, the mean local delay which is the average number of transmission attempts needed until the first successful target detection. For a given target distance, we obtain the optimal transmit probability that maximizes the density of successful radar detection while keeping the mean local delay below a threshold. We also provide several system design insights in terms of the fraction of reliable radar links, transmission delay, the density of vehicles, and congestion control.
\end{abstract}

\section{INTRODUCTION}

\section{A. Motivation}

Automotive radar is a key technology in modern vehicles that is used to detect the presence of nearby objects and proximal vehicles [1], [2]. These radars are now being widely used in advanced driver-assistance systems (ADAS) to warn the drivers of potential accidents. Due to such a widespread use of radars and shared spectrum, the mutual radar interference between vehicles is a rising concern. For instance, the vehicles traveling from the opposite direction can blind a radar, which might deteriorate the radar detection performance.

To accurately characterize the performance of an automotive radar system, it is critical to take into account the randomly located clutter in the environment [3] and the interfering vehicles [4]. In particular, while detecting the target vehicle, the radar observes reflections from the clutter resulting in ghost targets, which deteriorates the estimation efficiency. On the contrary, in the EU project MOSARIM [5], it is experimentally discovered that the signals from interfering vehicles are unlikely to cause ghost targets, but rather they create noiselike combined interference. Accordingly, the characterization of the interference and subsequently the signal-to-interferenceand-noise ratio (SINR) coverage is an important element in the system-level performance of vehicular radars. Although the topic of radar interference has been well-studied, many

This work has received funding from the European Research Council (ERC) under the European Union's Horizon 2020 research and innovation programme grant agreement number 788851 . works are based on simulation and empirical approaches that investigate the radar interference with complex ray-tracing and stochastic environments. Such approaches are not amenable to a link-level analysis of the radar detection performance, namely, the performance of individual radars, especially in the presence of dynamic wireless environment and traffic scenarios. In this paper, we aim to provide a fine-grained analysis of radar detection performance and give crisp insights into the system design.

\section{B. Related work}

In [6], authors present a simulation study to predict the interference potential between two radars, where the vehicles are distributed on a long street according to the traffic flow simulation. In [7], authors study the desired-to-undesired signal power ratio in ultra wideband automotive radar systems using the mix of analysis and simulations. The work in [8] studies the same metric by assuming a frequency modulation continuous wave (FMCW) scheme. These works, however, ignore different traffic scenarios due to dynamically changing vehicle density and locations with geographical regions and times. Such a randomness in the locations of vehicles can be captured using tools from stochastic geometry, where the locations of vehicles are modeled according to a point process [9]-[12].

For automotive radars, using stochastic geometry, [13] calculates the statistics of the interference power and the average SINR performance of vehicular radars for a highway scenario. For a planar geometry, [14] considers the strongest interferer approximation to obtain the radar detection range and the false alarm rate. While the previous works assume a constant radar cross-section (RCS), [15] have recently studied the standard successful radar detection probability $p_{\mathrm{S}} \triangleq \mathbb{P}(\operatorname{SINR}>\beta)$ with random RCS characterized by Swerling I model [16] that captures the varying physical features of the target vehicle. While $p_{\mathrm{s}}$ is an important performance metric, it is simply a spatial average of the detection performance of all radars in a given network realization. Hence, it does not reveal the performance of individual radars, which is of utmost importance from the safety point-of-view. Hence, in this paper, we provide a fine-grained view of the radar performance. In particular, we calculate the meta distribution [17] of the SINR, which yields the fraction of reliable radar links and enables one to characterize the transmission delay as well. 


\section{Contributions}

- For a bidirectional traffic scenario, we provide an analytical expression of the exact meta distribution (MD) of the SINR for the random RCS characterized by Swerling I model. We also give an accurate approximation of the MD by the beta distribution.

- We give simple analytical expressions of the standard successful radar detection probability and the mean local delay which captures the transmission delay.

- We propose an optimization framework to calculate the transmit probability of a vehicular radar that maximizes the density of successful radar detection while keeping the mean local delay below a threshold. We also give a congestion-control interpretation of this optimization.

- For fixed and random target distances, we highlight systemic tradeoffs that reveal conditions for reliable and low-latency radar detection.

\section{System MODEL}

\section{A. Network geometry and channel model}

We model a highway scenario by two parallel streets separated by distance $L$. Without loss of generality, we perform our analysis from the perspective of the typical vehicle that is located at the origin. The target vehicle is located in front of the typical vehicle at distance of $R$. The typical vehicle experiences interference from vehicles traveling in the opposite direction. Let the beamwidth of the radar be $\theta$. Thus only the vehicles beyond a minimum distance $\delta_{0}=\frac{L}{\tan \frac{\theta}{2}}$ from the origin are the interfering vehicles.

The locations of interfering vehicles are modeled as points of a 1-D Poisson point process (PPP) $\Phi \in \mathbb{R}$ of density $\lambda$. Let the coordinates of potential interfering vehicles be $z=(x, L) \in \Phi$. Let $p$ denote the probability that a vehicle transmits in a time slot. Then a point $z \in \Phi$ is marked as:

$$
K(z)= \begin{cases}0 & : x<\delta_{0} \\ \mathcal{B}(p) & : x \geq \delta_{0}\end{cases}
$$

where the vehicles closer than the distance $\delta_{0}$ do not interfere with the typical vehicle, and $\mathcal{B}(p)$ is the Bernoulli random variable with parameter $p$. The set $\mathcal{C}$ of interferers can be obtained by applying random marks to $\Phi$ and is given as $\mathcal{C}=$ $\{z: z \in \Phi, K(z)=1\}$.

The radar detection at the typical vehicle is considered to be successful if the received SINR is larger than a threshold $\beta$. We formulate the SINR in the following subsection.

\section{B. Channel model and SINR}

Let the transmit power of radars be $P$, and the transmit and receive antenna gains be $G_{\mathrm{t}}$ and $G_{\mathrm{r}}$, respectively. We adopt the standard path-loss model with path-loss exponent $\alpha$. Due to the wide variety of target vehicles, the target is assumed to have a fluctuating (random) RCS, $\sigma_{\mathrm{c}}$, which is an exponential random variable with mean $\bar{\sigma}$ based on the Swerling 1 model [16].

Let the effective area of aperture be $A_{\mathrm{e}}=\frac{G_{\mathrm{r}}}{4 \pi}\left(\frac{c}{f_{\mathrm{c}}}\right)^{2}$ with $c$ the speed of light and $f_{\mathrm{c}}$ the center frequency. Due to the lineof-sight, we assume that the desired signal does not suffer from any multipath and fading [13]. Consequently, the reflected signal power from the target vehicle reaches the typical vehicle with signal-strength [18]

$$
S=\gamma_{1} \sigma_{\mathrm{c}} P R^{-2 \alpha},
$$

where $\gamma_{1}=\frac{G_{\mathrm{t}}}{(4 \pi)^{2}} A_{\mathrm{e}}$. The interference is caused due to transmitted radar signals from $z \in \mathcal{C}$. These signals experience fast fading, which is modeled as Rayleigh with variance 1, i.e., the fading power is exponentially distributed with mean 1 . The interference at the typical vehicle is given by

$$
I=\sum_{z \in \Phi} \gamma_{2} P h_{z}\|z\|^{-\alpha} \mathbf{1}(z \in \mathcal{C}),
$$

where $\gamma_{2}=\frac{G_{\mathrm{t}} A_{\mathrm{e}}}{4 \pi}, h_{z}$ is the fading power, and $\mathbf{1}(\cdot)$ is the indicator function.

The SINR at the typical vehicle is given by

$$
\operatorname{SINR}=\frac{S}{N_{0}+I}=\frac{\gamma_{1} \sigma_{\mathrm{c}} P R^{-2 \alpha}}{N_{0}+\sum_{z \in \Phi} \gamma_{2} P h_{z}\|z\|^{-\alpha} \mathbf{1}(z \in \mathcal{C})},
$$

where $N_{0}=N_{\mathrm{d}} W$ is the noise power with $N_{\mathrm{d}}$ the noise power density and $W$ the bandwidth.

\section{Meta distribution (MD) of the SINR}

In a stationary and ergodic point process setting, the SINR MD provides a fine-grained view of the network. For instance, it permits the calculation of the fraction of reliable links in the network. Specifically, for an SINR threshold $\beta$ and a reliability threshold $t$, the meta distribution of the SINR is defined as

$$
\bar{F}_{P_{\mathrm{s}}}(\beta, t) \triangleq \mathbb{P}\left(P_{\mathrm{s}}(\beta)>t\right), \quad \beta \in \mathbb{R}^{+}, t \in[0,1],
$$

where the random variable $P_{\mathrm{s}}(\beta)$ represents the link reliability (or, alternatively, link success probability) and is given as

$$
P_{\mathrm{S}}(\beta) \triangleq \mathbb{P}(\operatorname{SINR}>\beta \mid \Phi) \text {. }
$$

Here, conditioned on the point process $\Phi$, the probability is evaluated by averaging over the fading and the channel access scheme (ALOHA in our case). For any ergodic point process of vehicles, the MD can be interpreted as the fraction of vehicles that achieve an SINR of $\beta$ with reliability at least $t$ in each realization of the network. Note that the mean of $P_{\mathrm{S}}(\beta)$ is the standard success probability $p_{\mathrm{s}}(\beta)$ of radar detection, which is also an important metric and is given as

$$
p_{\mathrm{s}}(\beta) \triangleq \mathbb{P}(\operatorname{SINR}>\beta)=\mathbb{E}\left(P_{\mathrm{S}}(\beta)\right) \text {. }
$$

The MD has an extremely useful interpretation as the transmission delay, which is discussed in the following subsection.

\section{Mean Local Delay}

The mean local delay is an important performance metric that characterizes the transmission delay, namely, the mean number of transmission attempts needed until the first successful target detection in the presence of interference and noise [19]. In each transmission attempt, the typical vehicle transmits with probability $p$, and the transmission is successful with probability $P_{\mathrm{S}}(\beta)$ conditioned upon $\Phi$. Thus the local delay is a geometric random variable with the conditional 
mean $\frac{1}{p P_{\mathrm{s}}}$ (obtained averaging over the fading and ALOHA). Subsequently, the mean local delay $\mathcal{D}$ is given by

$$
\mathcal{D}=\mathbb{E}\left[\frac{1}{p P_{\mathrm{S}}}\right]=\frac{1}{p} \mathbb{E}\left[\frac{1}{P_{\mathrm{s}}}\right]=\frac{1}{p} M_{-1},
$$

where $M_{-1}$ is the -1 st moment of the distribution of $P_{\mathrm{s}}$, i.e., of the meta distribution.

\section{iII. Calculation of the Meta Distribution}

Lemma 1. Conditioned on $\Phi$, the success probability $P_{\mathrm{s}}(\beta)$ of radar detection is given by

$$
P_{\mathrm{S}}(\beta)=\mathcal{T}(R)\left(\prod_{z \in \Phi: x>\delta_{0}} \frac{p}{1+\frac{4 \pi \beta\|z\|^{-\alpha}}{\bar{\sigma} R^{-2 \alpha}}}+1-p\right),
$$

where $\mathcal{T}(R)=\exp \left(\frac{-\beta N_{0}}{\bar{\sigma} \gamma_{1} P R^{-2 \alpha}}\right)$.

Proof: The conditional success probability $P_{\mathrm{S}}$ is

$$
\begin{aligned}
& P_{\mathrm{S}}(\beta)=\mathbb{P}(\operatorname{SINR} \geq \beta \mid \Phi) \\
& =\mathbb{P}\left(\sigma_{\mathrm{c}}>\frac{\beta\left(N_{0}+\sum_{z \in \Phi} \gamma_{2} P h_{z}\|z\|^{-\alpha}\right) \mathbf{1}(z \in \mathcal{C})}{\gamma_{1} P R^{-2 \alpha}} \mid \Phi\right) \\
& \stackrel{(a)}{=} \mathcal{T}(R) \mathbb{E}\left(\exp \left(\frac{-\beta \sum_{z \in \Phi} \gamma_{2} h_{z}\|z\|^{-\alpha} \mathbf{1}(z \in \mathcal{C})}{\bar{\sigma} \gamma_{1} R^{-2 \alpha}}\right)\right) \\
& \stackrel{(b)}{=} \mathcal{T}(R)\left(\prod_{z \in \Phi: x>\delta_{0}} p \mathbb{E} \exp \left(\frac{-\beta \gamma_{2} P h_{z}\|z\|^{-\alpha}}{\bar{\sigma} \gamma_{1} P R^{-2 \alpha}}\right)+1-p\right) .
\end{aligned}
$$

The step $(a)$ is due to the exponential distribution of $\sigma_{c}$. The step $(b)$ is obtained by averaging over ALOHA and due to the fact that the fading is independent across all the interfering links. Finally, the expression in (7) follows from the Laplace transform of the exponentially distributed $h_{z}$.

The direct evaluation of the exact distribution of $P_{\mathrm{s}}$ is impossible. Thus, we take an indirect approach to evaluate it through the calculation of its moments.

Theorem 1. The b-th moment $M_{b}$ of $P_{\mathrm{s}}$ is given by

$$
M_{b}=\mathcal{T}^{\prime}(b, R) \exp \left(\lambda F_{b}\right), \quad b \in \mathbb{C},
$$

where

$$
\begin{gathered}
F_{b}=\sum_{k=1}^{\infty}\left(\begin{array}{l}
b \\
k
\end{array}\right) p^{k} \int_{x=\delta_{o}}^{\infty}\left(\frac{-\beta^{\prime}\left(x^{2}+L^{2}\right)^{-\alpha / 2}}{1+\beta^{\prime}\left(x^{2}+L^{2}\right)^{-\alpha / 2}}\right)^{k} \mathrm{~d} x, \\
\beta^{\prime}=\frac{4 \pi \beta}{\bar{\sigma} R^{-2 \alpha}}, \text { and } \mathcal{T}^{\prime}(b, R)=\exp \left(\frac{-b \beta N_{0}}{\bar{\sigma} \gamma_{1} P R^{-2 \alpha}}\right) .
\end{gathered}
$$

Proof: We have

$$
\begin{aligned}
M_{b}= & \mathbb{E}_{\Phi}\left(\mathcal{T}(R)\left(\prod_{z \in \Phi: x>\delta_{0}} \frac{p}{1+\frac{4 \pi \beta\|z\|-\alpha}{\bar{\sigma} R^{-2 \alpha}}}+1-p\right)\right)^{b} \\
= & \mathcal{T}^{\prime}(b, R) \mathbb{E}_{\Phi}\left(\prod_{z \in \Phi: x>\delta_{0}}\left(\frac{p}{1+\frac{4 \pi \beta\|z\| \|^{-\alpha}}{\bar{\sigma} R^{-2 \alpha}}}+1-p\right)^{b}\right) \\
= & \mathcal{T}^{\prime}(b, R) \exp \left(-\lambda \int_{\delta_{o}}^{\infty} 1-\right. \\
& \left.\left(\frac{p}{1+\beta^{\prime}\left(x^{2}+L^{2}\right)^{-\alpha / 2}}+1-p\right)^{b} \mathrm{~d} x\right) .
\end{aligned}
$$

Using the binomial expansion, (8) follows.

Some useful corollaries of Theorem 1 follow immediately.

Corollary 1. For the special case of $\alpha=2$, the first moment $M_{1}$ of $P_{\mathrm{s}}$ is given in the closed form as

$$
\begin{aligned}
p_{\mathrm{s}}(\beta) & =M_{1}=\exp \left(\frac{-\beta N_{0} R^{4}}{\bar{\sigma} \gamma_{1} P}\right) \exp \left(-p \lambda \frac{\beta^{\prime}}{\sqrt{\beta^{\prime}+L^{2}}}\right. \\
& \left.\times\left(\frac{\pi}{2}-\arctan \left(\frac{\delta_{0}}{\sqrt{\beta^{\prime}+L^{2}}}\right)\right)\right) .
\end{aligned}
$$

Corollary 2. The mean local delay is evaluated as

$$
\begin{aligned}
\mathcal{D}(p) & =\frac{1}{p} M_{-1}=\frac{1}{p} \exp \left(\frac{\beta N_{0}}{\bar{\sigma} \gamma_{1} P R^{-2 \alpha}}\right) \\
& \times \exp \left(\lambda \int_{\delta_{0}}^{\infty} \frac{p \beta^{\prime}\left(x^{2}+L^{2}\right)^{-\frac{\alpha}{2}}}{1+(1-p) \beta^{\prime}\left(x^{2}+L^{2}\right)^{-\frac{\alpha}{2}}}\right) .
\end{aligned}
$$

For the special case of $\alpha=2$, (11) simplifies to

$$
\begin{aligned}
\mathcal{D}(p)= & \frac{1}{p} \exp \left(\frac{\beta N_{0} R^{4}}{\bar{\sigma} \gamma_{1} P}\right) \exp \left(p \lambda \frac{\beta^{\prime}}{\sqrt{(1-p) \beta^{\prime}+L^{2}}}\right. \\
& \left.\times\left(\frac{\pi}{2}-\arctan \left(\frac{\delta_{0}}{\sqrt{(1-p) \beta^{\prime}+L^{2}}}\right)\right)\right) .
\end{aligned}
$$

The exact meta distribution of the SINR can be calculated using the Gil-Palaez theorem as [17]

$$
F_{P_{\mathrm{s}}}(z)=\frac{1}{2}+\frac{1}{\pi} \int_{0}^{\infty} \frac{\Im\left(e^{-j u \log (z)} M_{j u}\right)}{u} \mathrm{~d} u,
$$

where $M_{j u}$ is the $j u$-th moment evaluated using (8).

\section{A. Approximation by the Beta Distribution}

Here we provide a simple yet accurate approximation of the SINR MD using the beta distribution. This approximation arises naturally as the support of $P_{\mathrm{S}}(\beta)$ is $[0,1]$. The MD can be approximated as the beta distribution by matching the first moment $M_{1}$ and the second moment $M_{2}$. Specifically, the MD can be approximately expressed as

$$
\bar{F}(\beta, t) \approx 1-I_{\varepsilon}\left(\frac{k_{1} k_{2}}{1-k_{1}}, k_{2}\right),
$$

where $I_{\varepsilon}(x, y)=\int_{0}^{1-\varepsilon} z^{x-1}(1-z)^{y-1} \mathrm{~d} z / B(x, y)$ is the regularized incomplete beta function with $B(\cdot, \cdot)$ the beta function, $k_{1}=M_{1}$, and $k_{2}=\left(M_{1}-M_{2}\right)\left(1-M_{1}\right) /\left(M_{2}-M_{1}^{2}\right)$. The accuracy of the beta approximation is confirmed in Fig. 1a.

\section{Optimal Transmission Probability}

Using first moment $M_{1}=p_{\mathrm{s}}$ (given by (5)), one can determine the density $d_{\mathrm{s}}$ of vehicles that observe a successful radar detection for a fixed target distance $R$. Specifically, $d_{\mathrm{s}}=p \lambda p_{\mathrm{s}}$. The operator prescribes an optimal transmit probability that maximizes $d_{\mathrm{s}}$ subject to a constraint on the mean local delay $\mathcal{D}$. In other words,

$$
\begin{aligned}
& p^{*}(R)=\underset{p \in(0,1]}{\arg \max } \quad d_{\mathrm{s}}(p)=p \lambda p_{\mathrm{s}} \\
& \text { subject to } \mathcal{D}(p)=\frac{1}{p} M_{-1} \leq d_{0}, \quad d_{0} \in[1, \infty) .
\end{aligned}
$$


The Karush-Kuhn-Tucker (KKT) conditions with the multiplier $\mu$ are as follows:

$$
\begin{aligned}
& \frac{\mathrm{d} \mathcal{L}}{\mathrm{d} p}=\frac{\mathrm{d}}{\mathrm{d} p}\left[p \lambda \exp \left(\frac{-\beta N_{0} R^{4}}{\bar{\sigma} \gamma_{1} P}\right) \exp \left(-p \frac{\lambda \pi \beta^{\prime}}{2 \sqrt{\beta^{\prime}+L^{2}}}\right)+\right. \\
& \left.\mu\left(\frac{1}{p} \exp \left(\frac{\beta N_{0} R^{4}}{\bar{\sigma} \gamma_{1} P}\right) \exp \left(\frac{p \pi \lambda \beta^{\prime}}{2 \sqrt{(1-p) \beta^{\prime}+L^{2}}}\right)\right)-d_{0}\right]=0 \\
& \mu\left[\frac{1}{p} \exp \left(\frac{\beta N_{0} R^{4}}{\bar{\sigma} \gamma_{1} P}\right) \exp \left(\frac{p \pi \lambda \beta^{\prime}}{2 \sqrt{(1-p) \beta^{\prime}+L^{2}}}\right)-d_{0}\right]=0 \\
& \mu \geq 0, \\
& \frac{1}{p} \exp \left(\frac{\beta N_{0} R^{4}}{\bar{\sigma} \gamma_{1} P}\right) \exp \left(\frac{p \pi \lambda \beta^{\prime}}{2 \sqrt{(1-p) \beta^{\prime}+L^{2}}}\right)-d_{0} \leq 0
\end{aligned}
$$

where (14) is the stationarity condition and (15) is the complementary slackness. Additionally, (16) and (17) are the dual and primal feasibility conditions.

Remark 1. For no constraint on $\mathcal{D}$, the optimal probability $p_{M}^{*}$ is obtained by equating $\frac{\mathrm{d} d_{\mathrm{s}}}{\mathrm{d} p}$ to 0 . For $\delta_{0}=0$, we have

$$
p_{M}^{*}(R)=\operatorname{clip}\left(\frac{2 \sqrt{\beta^{\prime}+L^{2}}}{\pi \lambda \beta^{\prime}}\right),
$$

where clip $(\cdot)$ is defined as

$$
\operatorname{clip}(x)= \begin{cases}0, & x<0 \\ x, & 0 \leq x \leq 1 \\ 1, & x>1\end{cases}
$$

Next, let $p_{1}$ and $p_{2}$ be the two solutions to the equation:

$$
\frac{1}{p} \exp \left(\frac{\beta N_{0} R^{4}}{\bar{\sigma} \gamma_{1} P}\right) \exp \left(\frac{p \pi \lambda \beta^{\prime}}{2 \sqrt{(1-p) \beta^{\prime}+L^{2}}}\right)=d_{0}
$$

From the complementary slackness condition, when $\mu=0$, the optimal transmit probability $p^{*}$ is evaluated as $p_{M}^{*}$ if $\frac{1}{p_{M}^{*}} \mathcal{D}\left(p_{M}^{*}\right)<d_{0}$. In other words, if the solution to (18) satisfies the delay constraint, the typical vehicle selects the same probability for a channel access. Also (as discussed in Fig. 3a), there exists two solutions ( $p_{1}$ and $p_{2}$, with $p_{1} \leq p_{2}$ ) to (20). Thus, in the case $p_{M}^{*}(R)$ does not satisfy (17), the optimal transmit probability is the solution to (20), i.e., either $p_{1}$ or $p_{2}$ which minimizes $d_{\mathrm{s}}$. This is further discussed in Section VI with reference to Fig. 4.

\section{RANDOM TARGET DISTANCE}

For fixed target distance, from (8), we note that the standard success probability $p_{\mathrm{s}}$, i.e., $M_{1}$, decreases with an increase in the density $\lambda$ of interfering vehicles. However, it is intuitive to assume that as $\lambda$ increases, i.e., in the case of dense vehicular traffic, the target vehicle is also closer to the typical vehicle. In that case, it is important to characterize the moments by averaging over the locations of the target vehicle for a given $\lambda .{ }^{1}$ For that, we assume that the locations of vehicles in the

\footnotetext{
${ }^{1}$ This is equivalent to averaging over a random target distance.
}

TABLE I

Network Parameters [2], [20]

\begin{tabular}{|l|l|l|}
\hline Parameter & Symbol & Value \\
\hline Transmit power & $P$ & $10 \mathrm{dBm}$ \\
\hline Lane distance & $L$ & $10 \mathrm{~m}$ \\
\hline Mean RCS & $\bar{\sigma}$ & $30 \mathrm{dbsm}$ \\
\hline Path loss exponent & $\alpha$ & 2 \\
\hline Antenna gain & $G_{\mathrm{t}}=G_{\mathrm{r}}$ & $30 \mathrm{dBi}$ \\
\hline Center frequency & $f_{\mathrm{c}}$ & $76.5 \mathrm{GHz}$ \\
\hline Noise power density & $N_{\mathrm{d}}$ & $-174 \mathrm{dBm} / \mathrm{Hz}$ \\
\hline Bandwidth & $W$ & $25 \mathrm{kHz}$ \\
\hline Beamwidth & $\theta$ & $15^{\circ}$ \\
\hline
\end{tabular}

lane of the typical vehicle form a PPP $\chi$, which is independent of $\Phi$, but has the same density $\lambda$. Accordingly, the $b$-th moment $\bar{M}_{b}$ of the MD is obtained by averaging $M_{b}$ given by (8) over the locations of the target vehicle. Thus, we have

$$
\bar{M}_{b}=\mathbb{E}_{R}\left[\exp \left(\frac{-b \beta^{\prime} R^{2 \alpha} N_{0}}{\gamma_{1} P}\right) \exp \left(-\lambda F_{b}\right)\right]
$$

where the pdf of $R$ is given by

$$
f_{R}(r)=\lambda \exp (-\lambda r) .
$$

Corollary 3. We notice that, for $b=1$,

$$
\begin{aligned}
\frac{\mathrm{d} p \lambda \bar{M}_{1}}{\mathrm{~d} \lambda} & =p \bar{M}_{1}+p \lambda\left[\int_{0}^{\infty} K_{m} \exp \left(-\lambda\left(F_{1}+r\right)\right) d r\right. \\
& \left.-\int_{0}^{\infty} K_{m} \lambda\left(F_{1}+r\right) \exp \left(-\lambda\left(F_{1}+r\right)\right) d r\right],
\end{aligned}
$$

where $K_{m}=\exp \left(\frac{-\beta^{\prime} R^{2 \alpha} N_{0}}{\gamma_{1} P}\right)$. Note that we have $\frac{d \bar{M}_{1}}{d \lambda} \geq 0$ for $\lambda \rightarrow 0$ and $\frac{d M_{1}}{d \lambda} \leq 0$ for $\lambda \rightarrow \infty$. This indicates that the fraction of vehicular links that experience a successful radar detection increases with $\lambda$ as it increases from 0 since the target vehicle comes closer to the typical vehicle. On the other hand, for a large $\lambda$, the interference increases, thereby decreasing the density $p \lambda \bar{M}_{1}$ of successful radar detection.

\section{RESUlts AND Discussions}

A list of parameters and their default values are given in Table I.

\section{A. Illustrations of the meta distribution}

Fig. 1a depicts the fine-grained reliability performance of radar detection. It shows qualitatively that, for $R=15 \mathrm{~m}$ and $\beta=5 \mathrm{~dB}$, most vehicles perform a successful radar detection with $40 \%$ reliability (i.e., with probability 0.4 ), while virtually no vehicle can perform a successful radar detection with $80 \%$ reliability. Fig. 1a also shows that $60 \%$ of vehicles can perform a successful radar detection with $60 \%$ reliability at $\beta=5 \mathrm{~dB}$. Hence, the meta distribution permits an analysis on a per-vehicle basis, which is of great importance in vehicular networks. Moreover, Fig. 1a confirms the accuracy of the beta approximation of the MD.

As a function of the SINR threshold $\beta$, for fixed reliability threshold $t$, Fig. 1b shows that the value of $\beta$ can be obtained such that a certain fraction of vehicles have a minimum 


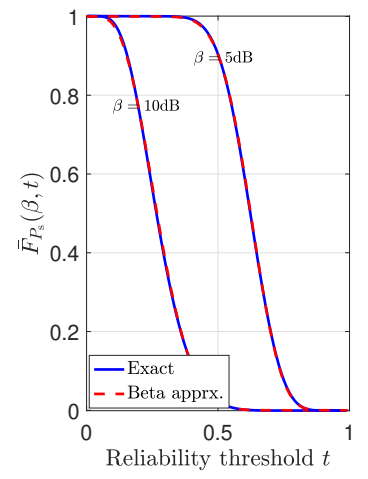

(a) The exact meta distribution and the beta distribution approximation for $R=15 \mathrm{~m}$.

Fig. 1. Illustrations of the meta distribution for $p=0.5, \lambda=0.04$.

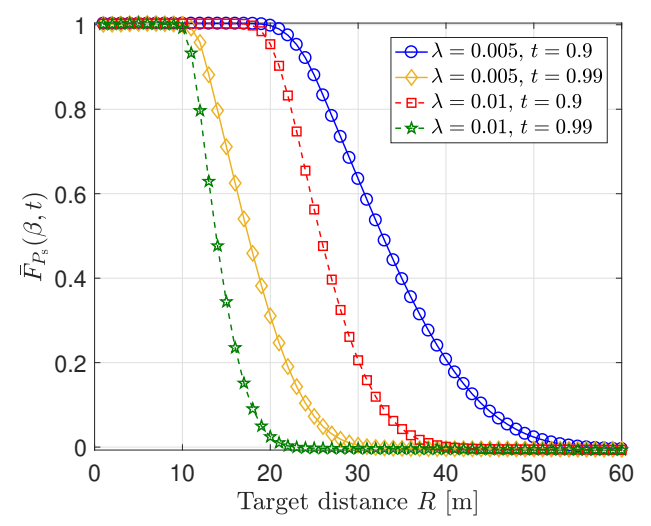

Fig. 2. Meta distribution as a function of fixed target distance $R$ at $p=0.2$ and $\beta=0 \mathrm{~dB}$ for different vehicle densities and reliability thresholds.

probability of successful radar detection. For instance, for fixed target distance, to achieve at least $99 \%$ probability of successful radar detection for $99 \%$ of the vehicles, an SINR threshold $\beta$ of at most $-14.96 \mathrm{~dB}$ may be chosen. Fig. 1b also depicts that a possibility of large $R$ due to its random nature leads to worse performance compared to the fixed target distance case at smaller $\beta$, while at a higher $\beta$, a possibility of small $R$ benefits the random distance case.

Fig. 2 depicts the ranging capability of the radar. Specifically, the maximum target distance to maintain a minimum probability of reliable radar detection can be determined for different $\lambda$ and $t$. For example, at $\lambda=0.005$, about $95 \%$ of vehicles can detect the target successfully with probability $99 \%$ for the maximum target distance of $20 \mathrm{~m}$.

\section{B. Mean local delay and density of successful radar detection}

For fixed target distance $R$, Fig. 3a shows the transmission delay performance of the radar through the mean local delay $\mathcal{D}$. In particular, a small transmit probability $p$ offers a smaller chance of transmission which increases the transmission period, while a high value of $p$ results in higher interference and hence reduces the probability of a successful radar detection.

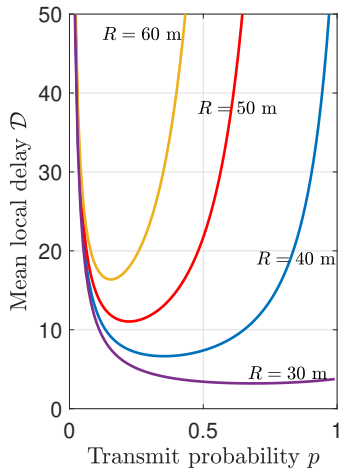

(a) Mean local delay against $p$.

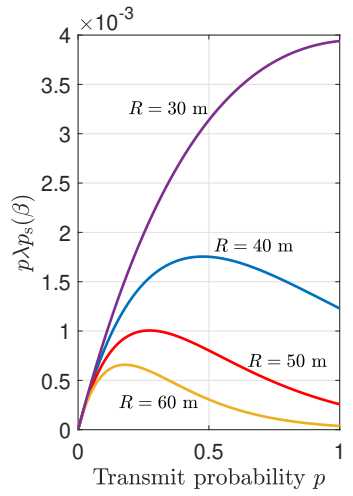

(b) Density of successful radar detection $d_{\mathrm{s}}=p \lambda p_{\mathrm{s}}$ against $p$.
Fig. 3. Tradeoffs associated with the transmit probability $p$ for different target distances $R$ at $\lambda=0.01$ and $\beta=0 \mathrm{~dB}$.

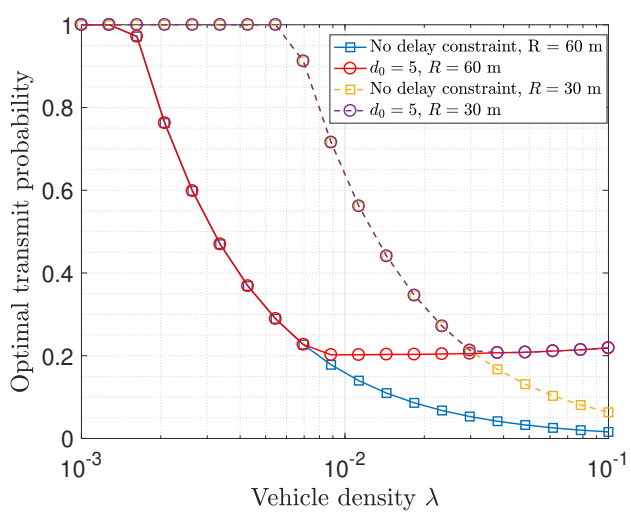

Fig. 4. Optimal transmit probability against $\lambda$ for different values of the target distance and delay constraints at $\beta=0 \mathrm{~dB}$.

Thus, for both cases, $\mathcal{D}$ increases. Also, an increase in $R$ reduces the successful radar detection probability, which increases $\mathcal{D}$. A complementary behavior with $p$ can be observed for the density $d_{\mathrm{s}}=p \lambda p_{\mathrm{s}}$ of successful radar detection, i.e., an increase in $p$ reduces the standard success probability $p_{\mathrm{s}}$ due to the increased interference. Thus there might exist an optimal $p$ that maximizes $d_{\mathrm{s}}$ depending on other network parameters.

Fig. 4 now jointly considers the effect of $p$ on $\mathcal{D}$ and $d_{\mathrm{s}}$. In particular, it shows the optimal transmit probability $p^{*}$ that maximizes the density $d_{\mathrm{s}}$, while keeping $\mathcal{D}$ below a threshold $d_{0}$. The probability $p^{*}$ is obtained by solving (13) for fixed target distance $R$ and vehicle density $\lambda$. This provides a framework for congestion control, where a channel is said to be congested when the number of vehicles accessing it goes beyond a threshold degrading the radar detection performance. One method to control the congestion is to reduce the access rate of vehicles, i.e., the transmit probability $p$ of vehicles.

For a small $\lambda$, the interference and hence the mean local delay is small. Thus $p^{*}$ is governed by (18) as if there is no delay constraint. On the contrary, as $\lambda$ increases beyond a threshold (e.g., $\lambda \geq 0.03$ for $R=30 \mathrm{~m}$ and $\lambda \geq 0.007$ for $R=60 \mathrm{~m}$ ) with $d_{0}=5, p^{*}$ does not decrease further. In this 


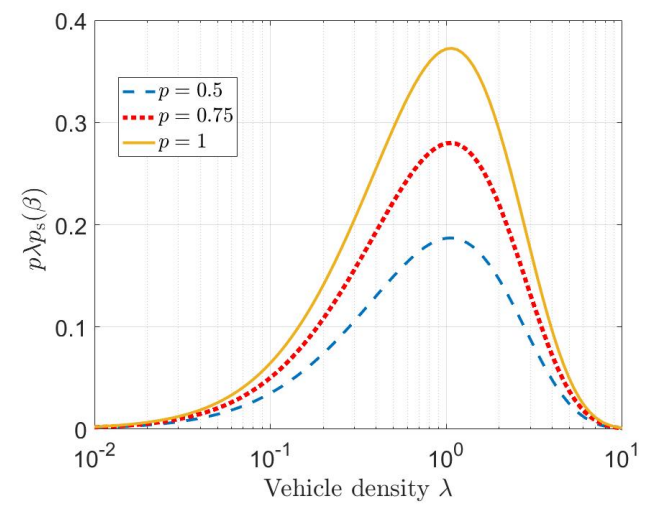

Fig. 5. The density $d_{\mathrm{S}}=p \lambda p_{\mathrm{S}}$ of successful radar detection against $\lambda . \beta=0$ dB.

regime, $p^{*}$ is governed by the solution to (20), and the typical vehicle accesses the channel more frequently to compensate for the reduced probability of successful radar detection due to increased interference with $\lambda$ and to satisfy the delay constraint of $d_{0}$. Note from Fig. 3a that two solutions to (20) satisfy a given delay constraint with equality, whereas, from (10), we see that $d_{\mathrm{s}}=p \lambda p_{\mathrm{s}}(\beta)$ is a function of the product of $p$ and $\lambda$. Consequently, for a high value of $\lambda$, the solution to (13) is the lower value of $p$ that satisfies (20).

Interestingly, for any $R, p^{*}$ is almost the same for a large $\lambda$. This is again evident from Fig. 3a where we see that, for a given $d_{0}$, different $R$ have the same $p_{1}$ (the lower value of $p$ that meets the delay constraint). Thus, for a large $\lambda$ with a delay constraint, the typical vehicle should transmit with a constant probability irrespective of the transmit distance.

For the random target distance $R$, Fig. 5 shows that there exists an optimal vehicle density $\lambda$ that maximizes the density $d_{\mathrm{s}}$ of successful radar detection, which is also shown in Corollary 3. Unlike the fixed target distance case, where $p^{*}$ can be less than 1 , for random $R, p=1$ maximizes $d_{\mathrm{s}}$.

\section{CONCLUSIONS}

The fine-grained reliability analysis of a vehicular radar using the meta distribution of the SINR offers several key design insights into the radar detection performance. For example, in the presence of radar interference and the random radar crosssection, our framework permits one to calculate the fraction of radars that perform a reliable target detection. Also, using this framework, one can calculate the mean local delay which characterizes the transmission delay. We provide performance limits of the reliable radar detection. In particular, one can determine the maximum SINR threshold, the maximum target distance, and the maximum vehicle density to allow a certain fraction of vehicles to perform reliable detection and satisfy the constraint on the maximum mean local delay. We also reveal the congestion-control aspect of our framework, where we show the behavior of the optimal transmit probability with the vehicle density to maximize the density of successful radar detection for both the fixed and random target distances. Specifically, the results show that, beyond a certain vehicle density, the optimal transmit probability increases with the density under stringent delay constraints.

From a broader perspective, since the reliability and the delay are two critical aspects of vehicular networks, the results in this work may contribute greatly towards understanding the ultrareliable low-latency communications (URLLC) in vehicular networks.

\section{REFERENCES}

[1] D. M. Grimes and T. O. Jones, "Automotive radar: A brief review," Proceedings of the IEEE, vol. 62, no. 6, pp. 804-822, 1974.

[2] J. Hasch, E. Topak, R. Schnabel, T. Zwick, R. Weigel, and C. Waldschmidt, "Millimeter-wave technology for automotive radar sensors in the $77 \mathrm{GHz}$ frequency band," IEEE Transactions on Microwave Theory and Techniques, vol. 60, no. 3, pp. 845-860, 2012.

[3] A. R. Chiriyath and D. W. Bliss, "Effect of clutter on joint radarcommunications system performance inner bounds," in Asilomar Conference on Signals, Systems and Computers (ASILOMAR), pp. 1379-1383, 2015.

[4] G. M. Brooker, "Mutual interference of millimeter-wave radar systems," IEEE Transactions on Electromagnetic Compatibility, vol. 49, no. 1, pp. 170-181, 2007.

[5] M. Kunert, "The EU project MOSARIM: A general overview of project objectives and conducted work,' in European Radar Conference, pp. 15, 2012.

[6] T. Schipper, S. Prophet, M. Harter, L. Zwirello, and T. Zwick, "Simulative prediction of the interference potential between radars in common road scenarios," IEEE Transactions on Electromagnetic Compatibility, vol. 57, no. 3, pp. 322-328, 2015.

[7] H. Kato and T. Kobayashi, "Detection probability of automotive radars using maximum length sequences to suppress interference from nearby radars," in IEEE National Aerospace and Electronics Conference, pp. 396-400, 2014.

[8] M. Goppelt, H.-L. Blöcher, and W. Menzel, "Analytical investigation of mutual interference between automotive FMCW radar sensors," in German Microwave Conference, pp. 1-4, 2011.

[9] B. Blaszczyszyn, P. Muhlethaler, and Y. Toor, "Stochastic analysis of ALOHA in vehicular ad hoc networks," Annals of Telecommunications, vol. 68, no. 1, pp. 95-106, 2013.

[10] C. Choi and F. Baccelli, "Poisson Cox point processes for vehicular networks," IEEE Transactions on Vehicular Technology, vol. 67, no. 10, pp. 10160-10165, 2018.

[11] V. V. Chetlur and H. S. Dhillon, "Success probability and area spectral efficiency of a VANET modeled as a Cox process," IEEE Wireless Communications Letters, vol. 7, no. 5, pp. 856-859, 2018.

[12] J. P. Jeyaraj and M. Haenggi, "Cox models for vehicular networks: SIR performance and equivalence," IEEE Transactions on Wireless Communications, vol. 20, no. 1, pp. 171-185, 2021.

[13] A. Al-Hourani, R. J. Evans, S. Kandeepan, B. Moran, and H. Eltom, "Stochastic geometry methods for modeling automotive radar interference," IEEE Transactions on Intelligent Transportation Systems, vol. 19, no. 2, pp. 333-344, 2017.

[14] A. Munari, L. Simić, and M. Petrova, "Stochastic geometry interference analysis of radar network performance," IEEE Communications Letters, vol. 22, no. 11, pp. 2362-2365, 2018.

[15] Z. Fang, Z. Wei, X. Chen, H. Wu, and Z. Feng, "Stochastic geometry for automotive radar interference with RCS characteristics," IEEE Wireless Communications Letters, pp. 1-1, 2020.

[16] D. Shnidman, "Expanded Swerling target models," IEEE Transactions on Aerospace and Electronic Systems, vol. 39, no. 3, pp. 1059-1069, 2003.

[17] M. Haenggi, "The meta distribution of the SIR in Poisson bipolar and cellular networks," IEEE Transactions on Wireless Communications, vol. 15, no. 4, pp. 2577-2589, 2016.

[18] D. P. Meyer and H. Meyer, "Review of the radar range equation," Radar Target Detection-Handbook of Theory and Practice, pp. 1-3, 1973.

[19] F. Baccelli and B. Błaszczyszyn, "A new phase transitions for local delays in MANETs," in IEEE INFOCOM, pp. 1-9, 2010.

[20] "Systems characteristics of automotive radars operating in the frequency band 76-81 GHz for intelligent transport systems applications, document ITU-R M.2057-0, ITU-R M series,” 2014. International Telecommunication Union. 